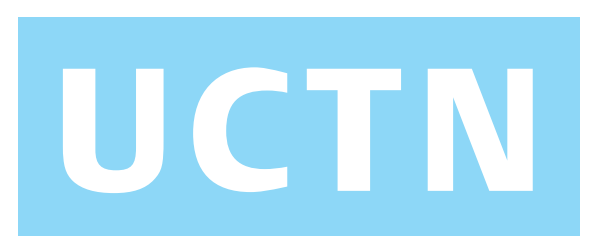

\title{
Gastric ulcer penetrating into the heart
}

A 57-year-old man developed hematemesis and was referred to our institution. Esophagogastroduodenoscopy showed that he had an active gastric ulcer, $15 \mathrm{~mm}$ in diameter, on the lesser curvature in the upper third of the corpus (Figure $\mathbf{1}$ ). The ulcer was generally elevated, as if it was being pushed up by an extragastric mass, and it had a pulse that was synchronous with the heartbeat. As the endoscopic findings suggested either the presence of a large artery at the ulcer base or penetration to the heart, emergency surgery rather than endoscopic therapy was planned for hemostasis. However, the patient suffered repeated massive hematemesis before surgery and went into hypovolemic shock. Unfortunately, he died despite vigorous attempts at cardiopulmonary resuscitation. At autopsy, a benign peptic ulcer, $15 \mathrm{~mm} \times 15 \mathrm{~mm}$ in size, was found on the lesser curvature in the upper third of the corpus, and this had penetrated through the pericardium and myocardium into the cardiac lumen at the apex of the heart (Figure 2 ). Histological examination revealed no evidence of malignancy and Helicobacter pylori was not detected.

Penetration of the pericardium and the heart is a rare complication of benign peptic ulcer, and was first described by Oser in 1880 [1]. The site of cardiac involvement determines the mode of presentation and the clinical course $[2,3]$. The cases that have been reported can be categorized into three groups according to the site of cardiac involvement: perforation into the pericardium, involvement of the left ventricle, and perforation into the atria. Generally, intra-abdominal gastric ulcers tend to perforate into the pericardium or the left ventricle, and early surgical intervention offers the only hope of survival. Although esophagogastroduodenoscopy is not helpful in terms of treatment, the endoscopic findings in the present case suggested penetration to the heart and might have aided early diagnosis if the patient had presented sooner. Unfortunately, such patients have invari-

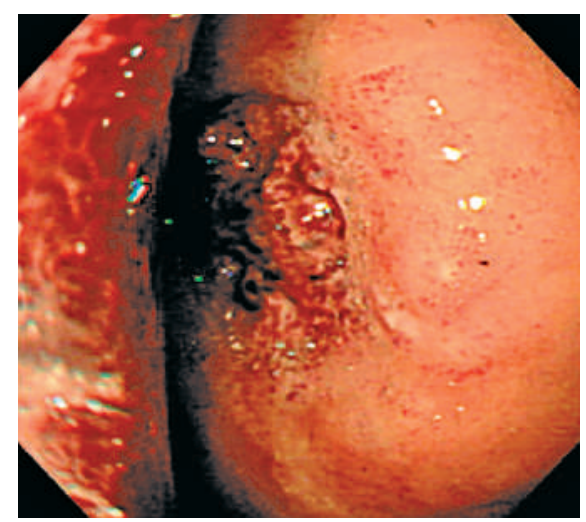

Figure 1 Esophagogastroduodenoscopy revealed an active gastric ulcer, $15 \mathrm{~mm}$ in diameter, on the lesser curvature in the upper third of the corpus.

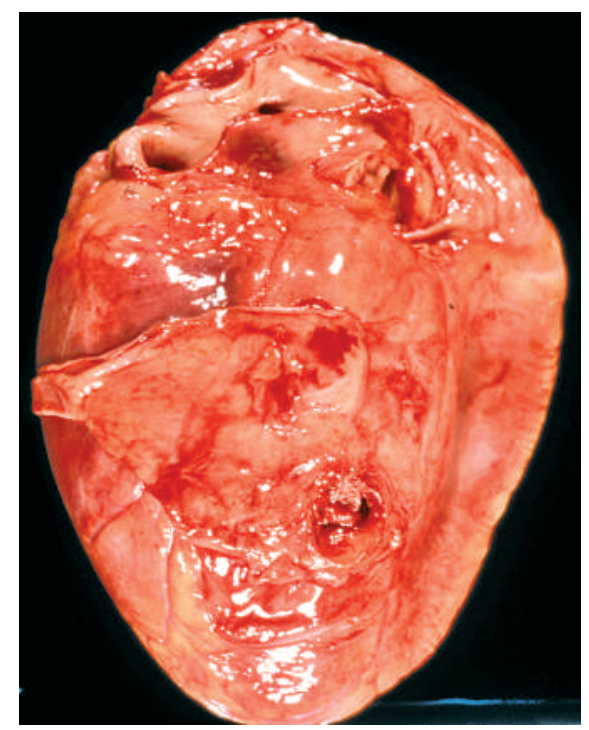

Figure 2 At autopsy, a benign peptic ulcer, $15 \mathrm{~mm} \times 15 \mathrm{~mm}$ in size, was found to have penetrated through the pericardium and myocardium into the cardiac lumen at the apex of the heart.

ably been reported to suffer massive and fatal hemorrhage.

\section{Acknowledgement}

This case was presented, in part, in Japanese at our own institute in the Dokkyo Medical Journal.
Endoscopy_UCTN_Code_CCL_1AB_2AD_3AC

Kuang-I Fu', H. Fukui', K. Tominaga², Y. Kaji ${ }^{3}$, H. Hiraishi ${ }^{2}$, T. Fujimori ${ }^{1}$

${ }^{1}$ Department of Surgical and Molecular Pathology, Dokkyo University School of Medicine, Tochigi, Japan

2 Department of Gastroenterology, Dokkyo University School of Medicine, Tochigi, Japan

${ }^{3}$ Department of Radiology, Dokkyo University School of Medicine, Tochigi, Japan.

\section{References}

${ }^{1}$ Finney JM. Ulcers of the stomach opening into the left ventricle of the heart. BMJ 1886; 1 : $1102-1104$

2 Porteous C, Williams D, Foulis A, Sugden BA. Penetration of the left ventricular myocardium by benign peptic ulceration: two cases and a review of the published work. J Clin Pathol 1984; 37: 1239-1244

${ }^{3}$ West AB, Nolan N, O'Brian DS. Benign peptic ulcers penetrating pericardium and heart: clinicopathological features and factors favoring survival. Gastroenterology 1988; 94: $1478-1487$

\section{Corresponding Author}

\section{Kuang-I Fu, M.D., Ph.D.}

Department of Surgical and Molecular Pathology

Dokkyo University School of Medicine 880 Kitakobayashi, Mibu

Shimotuga

Tochigi 321-0193

Japan

Fax: +81-282-86-5678

E-mail: fukuangi@hotmail.com

DOI: $10.1055 / \mathrm{s}-2006-944631$ 